\title{
A Review of Color Science in Dentistry: The Process of Color Vision
}

\author{
James C. Ragain* \\ Associate Professor, Department of Restorative Dentistry, University of Tennessee Health Sciences \\ Center, College of Dentistry, Memphis, TN, USA
}

Received: November 11, 2014; Accepted: February 25, 2015; Published: 10 March, 2015

*Corresponding author: Dr. James C. Ragain, Associate Professor, Department of Restorative Dentistry, College of Dentistry, University of Tennessee Health Sciences Center, Memphis, TN, USA, Tel: 901-448-1323; E-mail: jragain@uthsc.edu

\begin{abstract}
This article is one of a series review articles discussing the applications of color science to the clinical practice of dentistry. In this segment, the basic perception of color by humans, as well as, tests for color visual acuity is discussed.
\end{abstract}

\section{Introduction}

The primary goals of the restorative dentist are to restore the function of damaged or missing tooth structures and to maintain stability in the dentition. Damaged or dentition can result from congenital abnormalities, trauma, or disease. In some cases, patients are unhappy with the esthetic appearance of their teeth. For many patients, restoring dental esthetics is of the utmost importance, and they will ignore the value of function and emphasize the importance of appearance. For some, their overall feelings of well-being can be influenced by the appearance of their dentition. The blending of restorative materials with the teeth, producing a natural-looking appearance suitable to the patient and in which the dental restoratives cannot be seen, should be a major goal of the restorative dentist.

Current clinical techniques for the matching of natural tooth color with esthetic restorative materials and prostheses generally provide clinically acceptable results for the patients. However, shade guides with shade tabs are commonly used for shade selection and these techniques are very subjective, and often these techniques depend on numerous ambient factors and color vision acuity of the clinician. In the modern dental practice colorimetric instrumentation can be used to choose a close color match. However, the final acceptance of the color match rests with the human observer. Overall appearance is affected not only by color but also gloss, texture, cultural bias, ambient conditions, and personal tastes.

This article is the first of a series of articles that will review the fundamentals of color science as applied to the practice of dentistry. This first article will discuss the basic human perception of color. A review of the current accepted theory of human color perception will be discussed. Future articles will discuss color measurement, color difference calculations, and suggestions for shade selection in the dental practice. An understanding of the principles of color vision and how they are applied to clinical dentistry will enhance the restorative dentist's abilities to meet patient expectations in regards to the esthetics of dental restorations.

\section{The Human Perception of Color}

There are three distinct and common uses of the term color. In the field of chemistry, color is used as a generic term for dyes and pigments. In physics, it is used to refer to optical phenomena with measurement in terms of relevant optical properties of an object. In the life sciences, such as physiology and psychology, color is dealt with in terms of the human visual process and sensation in the consciousness of the human as an optical observer [1]. The combined intensities of the wavelengths present in a beam of visible light determine the property referred to as color. Visible light is a form of electromagnetic radiant energy in the wavelength range of approximately 400-700 nm (Figure 1) which can be detected by the human eye [2]. An object must either emit light, or it must reflect or transmit incident light from an external source for it to be visible. It can be said that the perception of color is the result of a physiological response to a physical stimulus [3].

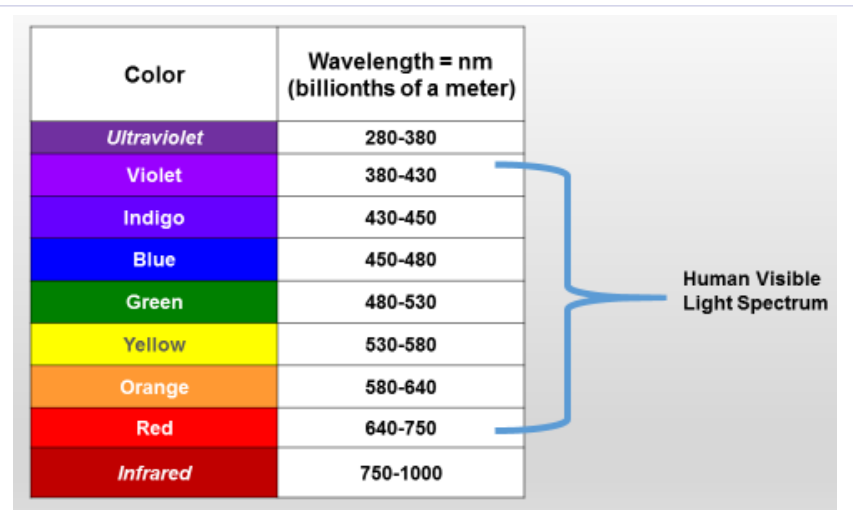

Figure 1: Human visible light spectrum. 
Light from an object that is incident on the eye is focused onto the retina by the corneal lens. The light is converted into nerve impulses which are subsequently transmitted to the cerebral cortex. The retina is made up of rod and cone-shaped cells. The cone cells are responsible for color vision. The six to seven million cones provide the eye's color sensitivity. The cones can be divided into "red" cones, "green" cones, and "blue" cones. The rod cells are monochromatic. They are responsible for our darkadapted vision. Rods are incredibly efficient photoreceptors. The rods are more numerous than cones, some 120 million, and are more sensitive than the cones to light. The light response of the rods peaks sharply in the blue, and they respond very little to red light [4]. Figure 2 is a microphotograph of stained rod and cone cells.

The cones are much more concentrated in the central yellow spot known as the macula. In the center of that region is the fovea centralis (red and green cones only), a $0.3 \mathrm{~mm}$ diameter rod-free area with very thin, densely packed cones. These cells exhibit a response curve related to the wavelength of the incident light.

The human perception of color depends on the physical parameters of light and a complex abstraction by brain neurons of the physical parameters of the reflectance of light from objects. The experience of color and the attributes of color given to objects is created by this abstraction. This is accomplished by discriminating between the wavelengths of light reflected from an object from those of light reflected from the environment.

As previously mentioned, the human eye is sensitive to wavelengths of light in the range of 400-700 $\mathrm{nm}$. Throughout this range of visible light there is a gradual change in color from blue, through green, yellow, orange, to red. The eye is most sensitive to light in the green-yellow region. In 1802, a three variable, or trichromatic theory of color vision was proposed. This theory is based upon the action of three differing receptors located in the retina. It was theorized that there is an overlapping of the absorption spectra from the three receptors, and excitation of each is transmitted independently to the brain. This theory subsequently received independent support in the mid-nineteenth century when it was demonstrated that all the colors perceived by humans can be matched by mixtures of three spectral lights. This theory was further promoted when the absorption spectra of pigments of single cones in the human eye were directly measured. Figure 3 is a concept map of the basic interactions of incident light from an object with the human eye. It was determined that the individual cones contain only one of three pigments. One pigment is primarily sensitive to short wavelengths in the visible spectrum and peaks at approximately 430-446 $\mathrm{nm}$ and is responsible for making a strong contribution to the color blue. Another pigment is sensitive to the middle wavelengths and peaks at $531-555 \mathrm{~nm}$. This pigment contributes to the color green. The third pigment peaks at 558-599 $\mathrm{nm}$ and contributes to the color red [3,4]. All of the colors in the human visible spectrum can be derived through the additive color theory (Figure 3).

Psychological experiments have produced results similar

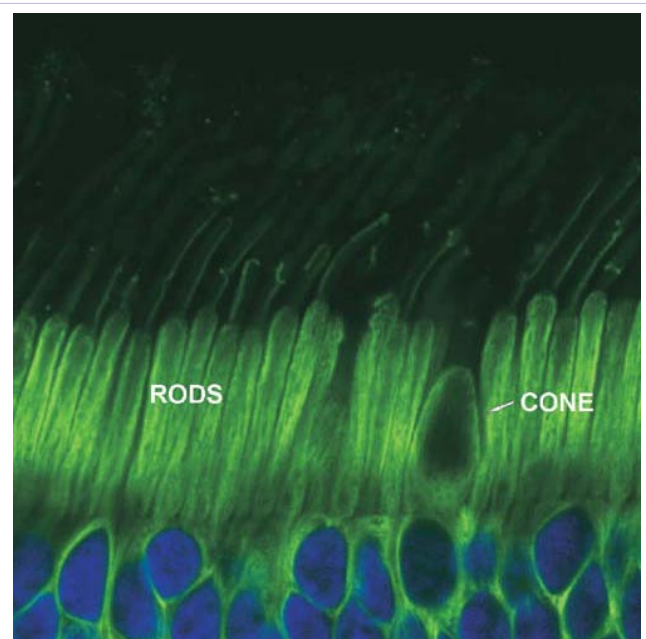

Figure 2: Microphotograph of stained rod and cone cells.
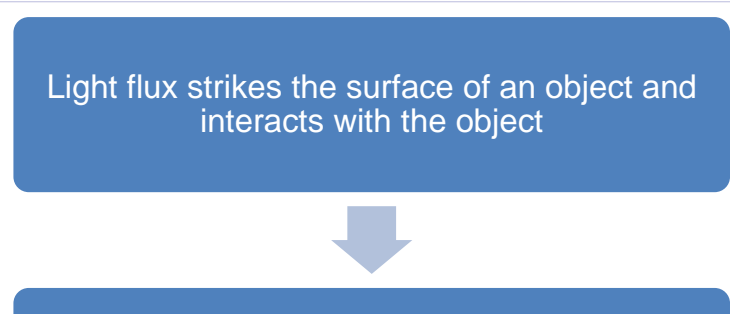

Light from the object that is incident on the eye is focused onto the retina by the corneal lens.
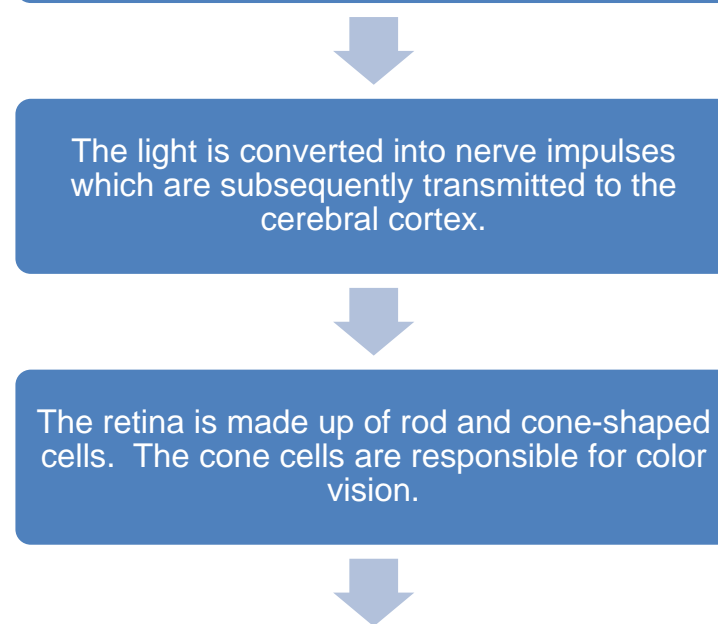

The human perception of color depends on the physical parameters of light and a complex abstraction by brain neurons of the physical parameters of the reflectance of light from objects. The experience of color and the

attributes of color given to objects is created by this abstraction.

Figure 3: Concept map of light interactions with the human eye. 
to those described above. These tests measured the spectral responses by using the subject's answers to visual stimuli. The best psychological data were obtained from human subjects who had genetic defects in their photoreceptors. Some of these patients had only two cone pigments, and they were described as dichromats. Others having only one cone pigment were classified as monochromats.

The trichromatic theory of color vision attributes color perception to the activity of three primary cone mechanisms. The message generated reaches the cortex in independent, parallel channels without significant intersection or blending of mutual elements. At the cortex the three outputs are compared for color vision. The trichromatic theory has found support in the discovery of three cone pigments capable of responding best to those parts of the spectrum that contribute to the human perception of blue, green and red.

Evidence for the trichromatic theory comes from color matching and color mixing studies. Young and Helmholtz [4] carried out experiments in which individuals adjusted the relative intensity of light sources of different wavelengths so that the resulting mixture field matched an adjacent test field composed of a single wavelength. Individuals with normal color vision needed three different wavelengths to match any other wavelength in the visible spectrum. This finding led to the hypothesis that normal color vision is based on the activity of three types of receptors, each with different peak sensitivity. Consistent with the trichromatic theory, we now know that the overall balance of activity in S (short wavelength), M (medium wavelength), and L (long wavelength) cones determines our perception of color as shown in Figure 4.

Trichromacy alone fails to explain a variety of color phenomena such as why certain color combinations cancel each other out. According to the opponent process theory, there are three primary color pairs coupled in a mutually antagonist fashion. These pairings are red-green, yellow-blue, and blackwhite.

The color opponent theory explains why certain colors within the boundaries of a perceived object cancel one another. It does not explain simultaneous color contrast, which is the

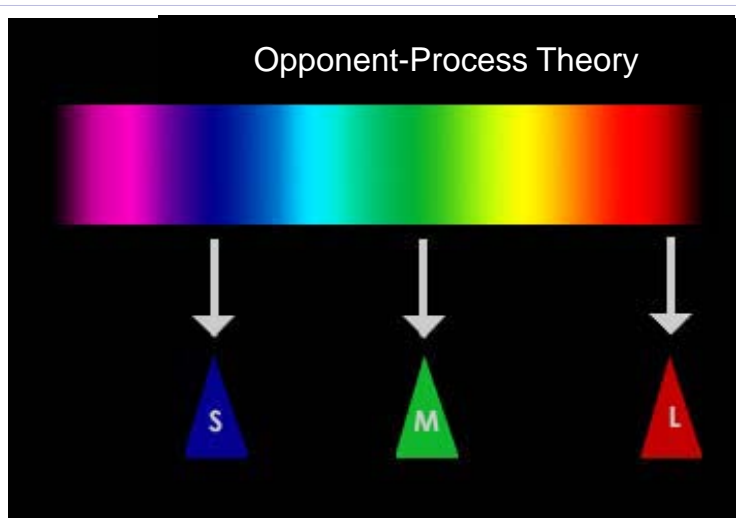

Figure 4: Trichromatic theory of color vision. phenomenon where different cone mechanisms appear to facilitate rather than inhibit one another. Color contrast occurs across rather than within the boundaries of a perceived object. An example would be a gray object on a red background is seen as having a green tinge, or when on a green background, it has a red tinge [3].

Developed by Ewald Hering [5] the opponent-process theory states that the cone photoreceptors are linked together to form the three opposing color pairs: blue/yellow, red/green, and black/white. Activation of one member of the pair inhibits activity in the other. Consistent with this theory, no two members of a pair can be seen at the same location, which explains why we don't experience such colors as "bluish yellow" or "reddish green". This theory also helps to explain some types of color vision deficiency. For example, people with dichromatic deficiencies are able to match a test field using only two primaries. Depending on the deficiency they will confuse either red and green or blue and yellow.

The opponent-process theory explains how we see yellow though there is no yellow cone. It results from the excitatory and inhibitory connections between the three cone types. Specifically, the simultaneous stimulation of red (L cones) and green (M cones) is summed and in turn inhibits $\mathrm{B}+\mathrm{Y}-$-, which results in the perception of yellow. However, when blue light is present, the $S$ cone is activated, the $\mathrm{B}+\mathrm{Y}$ - cell receives excitatory input and blue is perceived (Figure 6).

A combination of the opponent process and the trichromatatic theories is necessary to fully explain the perception of color. It has been proposed that color vision occurs in stages. The first stage is at the level of the cones. Here color vision is trichromatic. At subsequent stages transformation is made to opponent color form.

\section{Tests for Color Vision}

It is highly recommended that dentists, dental hygienists, and dental assistants have their color vision tested. There are several very good color vision screening tests including the American Optical Company Hardy-Rand-Ritter (AO H-R-R Test) (Southbridge, MA, USA) and the Ishihara Test for Color Deficiency (Tokyo, Japan). These screening tests use pseudo-isochromatic plates to determine if an individual has a color vision deficiency (Figure 4). The Farnsworth-Munsell 100 Hue Test (F-M 100 Hue Test) (Munsell Color, Macbeth, Division of Kollmorgen Corp, Baltimore, MD, USA) (Figure5) can be administered to quantify a person's color discrimination abilities. This test presents a relatively simple means of testing an individual's abilities to judge small color differences in objects. This test also measures the zones of color confusion of persons with defective color vision [6].

About $2 \%$ of the male population is red-green color blind because of a lack of the red or green pigment (conditions called protanopia and deuteranopia, respectively). The incidence in females is much lower because they are likely to have at least one $\mathrm{X}$ chromosome with normal red and green genes. Lack of the 


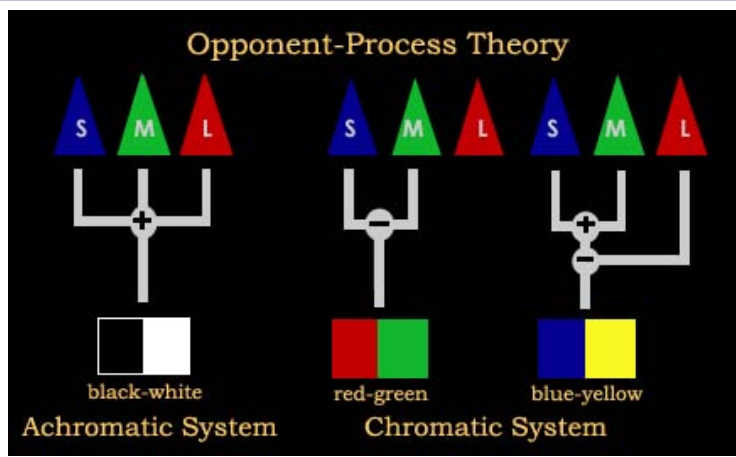

Figure 5: Opponent theory of color vision.

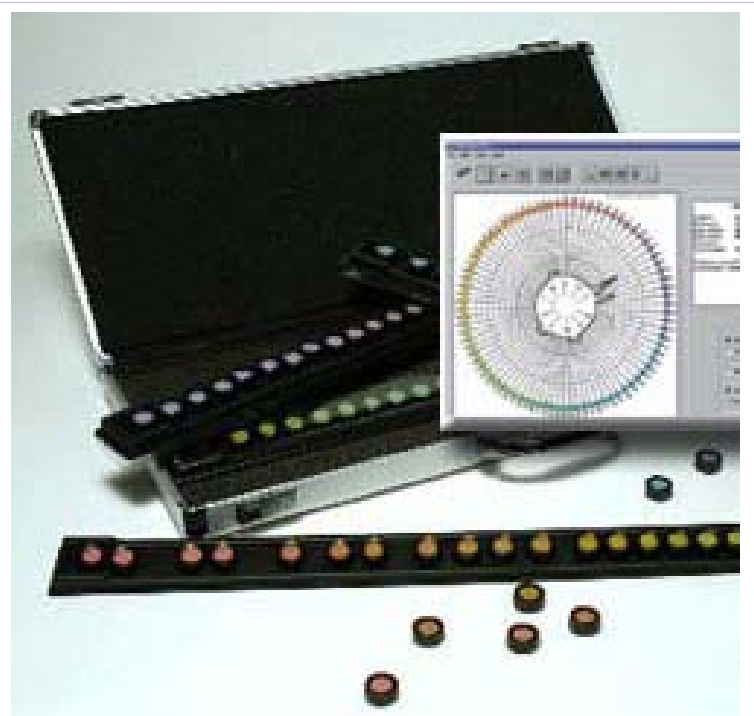

Figure 6: The Farnsworth-Munsell 100 Hue Test (F-M 100 Hue Test). blue cone pigment (tritanopia) is rare and is equally uncommon in males and females [7].

\section{Conclusion}

This article briefly described the complicated process of color perception by humans. Future review articles in this series will enlighten the dental clinician as to various aspects of color in the practice of esthetic dentistry.

\section{References}

1. MacAdams DL. Color measurement, theme and variations. IInd ed. New York: Springer-Verlag; 1985.

2. Judd DB and Wyszecki G. Color in business, science, and industry. IIIrd ed. New York: Wiley and Sons; 1975.

3. Kandel ER, Schwartz JH, Jessell TM, Siegelbaum SA, Hudspeth AJ. Princilpes of neural science. Vth ed. New York: McGraw-Hill Professional Publishing 2012.

4. Jacobs GH. The discovery of spectral opponency in visual systems and its impact on understanding the neurobiology of color vision. J Hist Neurosci. 2014; 23(3): 287-314.

5. Martin PR, Lee BB. Distribution and specificity of S-cone ("blue cone") signals in subcortical visual pathways. Vis Neurosci. 2014; 31(2): 17787

6. Nolte J. The Human Brain: An Introduction to Its Functional Anatomy, VIth ed. St. Louis: Mosby Vital Book file; 2009.

7. Ragain JC. Matching the Optical Properties of Direct Esthetic Dental Restorative materials to Those of Human Enamel and Dentin. Columbus: Hathi Trust Digital Library; 1998. 